\title{
Colitis Induced by Colon-Cleansing Agent
}

\author{
Shehriyar Mehershahi ${ }^{a}$ Haider Ghazanfar ${ }^{b}$ Shoaib Ashraf ${ }^{b}$ \\ Danial H. Shaikh ${ }^{a}$ Harish Patel $^{a}$ \\ ${ }^{a}$ Department of Gastroenterology, Bronxcare Health System, Bronx, NY, USA; ${ }^{b}$ Department \\ of Medicine, Bronxcare Health System, Bronx, NY, USA
}

\section{Keywords}

Irritable bowel syndrome $\cdot$ Constipation $\cdot$ Colitis $\cdot$ Colon-cleansing agent

\begin{abstract}
Constipation is one of the most common functional gastrointestinal disorders and affects $20 \%$ of the general population. Irritable bowel syndrome (IBS) is a functional disorder of the gastrointestinal tract that affects the large intestine and is characterized by chronic abdominal pain and altered bowel habits. We report a case of a 35-year-old African American man with a past medical history of IBS who presented to the clinic with a chief complaint of abdominal pain and bloody diarrhea for 1 week. The patient stated that he used a colon-cleansing agent because of persistent constipation. Computed tomography scan of the patient's abdomen and pelvis with contrast was performed which showed diffuse contiguous segmental mural thickening and nodularity seen along the distal transverse, descending, and sigmoid colon. Colonoscopy showed moderate diffuse inflammation characterized by altered vascularity, erythema, and granularity from the rectum to the descending colon, and localized mild inflammation characterized by erythema was found at the ileocecal valve. The patient's clinical condition improved with symptomatic management over 10 days. Patients with IBS should be advised to restrain from using a colon-cleansing agent without advice from their primary doctor as it can lead to various complications.
\end{abstract}

\section{Introduction}

Constipation is one of the most common functional gastrointestinal disorders affecting $20 \%$ of the general population [1]. Constipation is more prevalent in the elderly population. Elderly woman are 2-3 times more likely to suffer from constipation than their male counterparts [2].

\section{Karger!}


Irritable bowel syndrome (IBS) is a function disorder of the gastrointestinal tract that affects the large intestine and is characterized by chronic abdominal pain and altered bowel habits. According to a meta-analysis, the prevalence of IBS is around 11 percent [3]. IBS can be divided into 4 subtypes that include IBS with predominant constipation (IBS-C), IBS with predominant diarrhea, IBS with mixed bowel habits, and IBS unclassified.

The pathophysiology of IBS is uncertain and involves various factors [4]. Abnormalities in gastrointestinal motility and visceral hypersensitivity are considered the 2 major factors contributing to IBS pathophysiology [5, 6]. In diarrhea-predominant IBS, an exaggerated motor response is seen, while prolonged transit time is seen in IBS-C $[7,8]$. Colitis refers to the inflammation of the mucosal lining of colon. Symptoms of colitis include abdominal pain, cramping, fatigue, weight loss, diarrhea, urgency, rectal bleeding, and rarely incontinence. Common causes of colitis include inflammatory bowel disease (ulcerative colitis), ischemic colitis, pseudomembranous colitis, ischemic colitis, and microscopic colitis.

Rare causes of colitis include chemical colitis, nonsteroidal anti-inflammatory drugs (NSAIDs), and oral contraceptive-induced colopathy. One of the most common causes of chemical colitis is a result of exposure to glutaraldehyde during colonoscopy [9]. Mucosal injury resulting from chemical colitis can range from mild inflammation to severe ulceration and bleeding. The prevalence of NSAID-induced colopathy has been increasing due to the increased use of NSAID. The pathological manifestation of NSAID-induced colopathy includes inflammatory colitis, ulcers, strictures, ischemic colitis, eosinophilic colitis, and colonic hemorrhage [10]. Several over-the-counter medications are available for managing constipation in IBS. Enemas comprising hydrogen peroxide are commonly used as home remedies to treat constipation. Enema-containing hydrogen peroxides have been implicated in causing colitis [11]. We present a case of a 35-year-old with IBS, which developed colitis secondary to the use of a colon-cleansing agent with warm water.

\section{Case Report}

The patient is a 35-year-old African American man with a past medical history of IBS who presented to the clinic with a chief complaint of abdominal pain and bloody diarrhea for 1 week. The patient reported traveling from Florida to New York 2 weeks before the clinical symptoms. Ten days before admission, he started having constipation for which he ordered an online colon-cleansing enema (comprising hydrogen peroxide). The patient used the enema by mixing it with warm water. The patient successfully emptied his bowel without observing any blood in the initial bowel movements. The patient stated that he noticed rectal bleeding a few hours later. He started to have severe left lower quadrant pain of the abdomen which was cramping in nature. The patient graded the pain 10 on a scale of 1 to 10 . The patient denied any alleviating or aggravating factors. Patient had up to 4 bloody bowel movements per day for the last 1 week. In the clinic, he was dehydrated and was sent to the emergency department of our institution because of bloody diarrhea and the need for intravenous rehydration. In the emergency department, the patient had nausea and 1 episode of nonbloody nonbilious vomiting. On arrival at our hospital, he was alert and well oriented. His vital signs were as follows: heart rate 77 beats per minute, blood pressure 136/88 $\mathrm{mm} \mathrm{Hg}$, respiratory rate 15 times per minute, body temperature $36.5^{\circ} \mathrm{C}$, and oxygen saturation $98 \%$ in room air. A physical examination revealed moderate tenderness in his left lower abdomen without signs of peritonitis. Laboratory examination showed a hemoglobin level of $15.3 \mathrm{~g} / \mathrm{dL}$, leucocytes $5.3 \mathrm{k} / \mu \mathrm{l}$, CRP of $28.62 \mathrm{mg} / \mathrm{L}$, aspartate aminotransferase of $18 \mathrm{unit} / \mathrm{L}$, and alanine aminotransferase of 21 unit/L, with no other significant alterations. Computed tomography scan of the patient's abdomen and pelvis with contrast was performed which showed diffuse

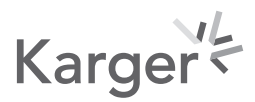


Fig. 1. CT scan of the patient's abdomen and pelvis with contrast was performed and showed diffuse contiguous segmental mural thickening and nodularity seen along the distal transverse, descending, and sigmoid colon (arrows). CT, computed tomography.

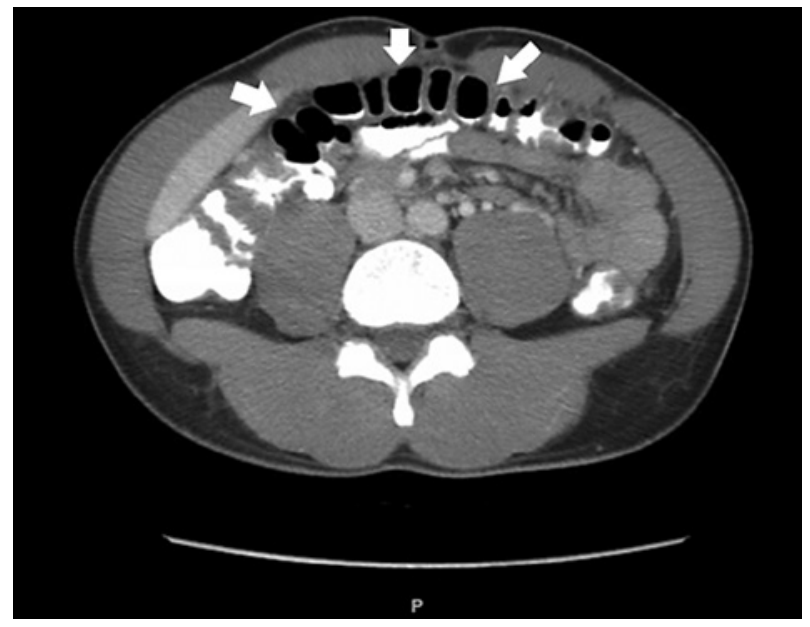

contiguous segmental mural thickening and nodularity seen along the distal transverse, descending, and sigmoid colon. There are multiple subcentimeter left colic mesenteric lymph nodes, which are likely reactive in etiology. This is shown in Figure 1.

Metronidazole, ceftriaxone, and intravenous rehydration were started. The gastroenterology division was consulted for possible colonoscopy. The patient consented for colonoscopy, and bowel preparation was done on the second day of admission. Colonoscopy showed moderate diffuse inflammation characterized by altered vascularity, erythema, and granularity from the rectum to the descending colon, and localized mild inflammation characterized by erythema was found at the ileocecal valve. This is shown in Figure 2.

Biopsies were taken with a cold forceps for histology. Ileocecal valve biopsy showed colonic mucosa with focal mild increased eosinophilic infiltrates in the lamina propria. Sigmoid colon biopsy showed colonic mucosa with mild superficial acute inflammation and focal acute cryptitis, no granuloma, crypt distortion, or basal plasmacytosis. Rectal biopsy showed colonic mucosa with mild superficial acute inflammation and focal acute cryptitis. The patient's clinical condition improved with symptomatic management over a course of 10 days.

\section{Discussion}

Various studies have shown that IBS has a detrimental impact on patients' quality of life $[12,13]$. Severe intestinal symptoms and psychological factors are associated with poor quality of life [14]. There is no difference seen in the quality of life among various IBS subtypes [13].

Lifestyle changes and dietary modifications are recommended in patients with mild symptoms of IBS. According to a randomized controlled trial, both a strict traditional IBS diet and a diet low in FODMAPs were associated with reduced IBS symptoms [15]. Patients with IBS should be encouraged to increase their physical activity as exercise has been associated with IBS improved intestinal symptoms [16].

Over-the-counter colon-cleansing agents are commonly used to treat constipation. Most of these agents are not FDA-approved and can lead to severe life-threatening complications. In our case, the patient developed colitis after using the colon-cleansing agent. Patients with IBS-C have a prolonged transit time, which can increase the colon wall exposure with the colon-cleansing agent, resulting in even more severe damage. According to a case report, inadequate removal of glutaraldehyde during a colonoscopy had a similar effect [17].

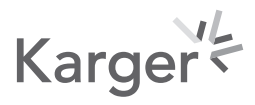



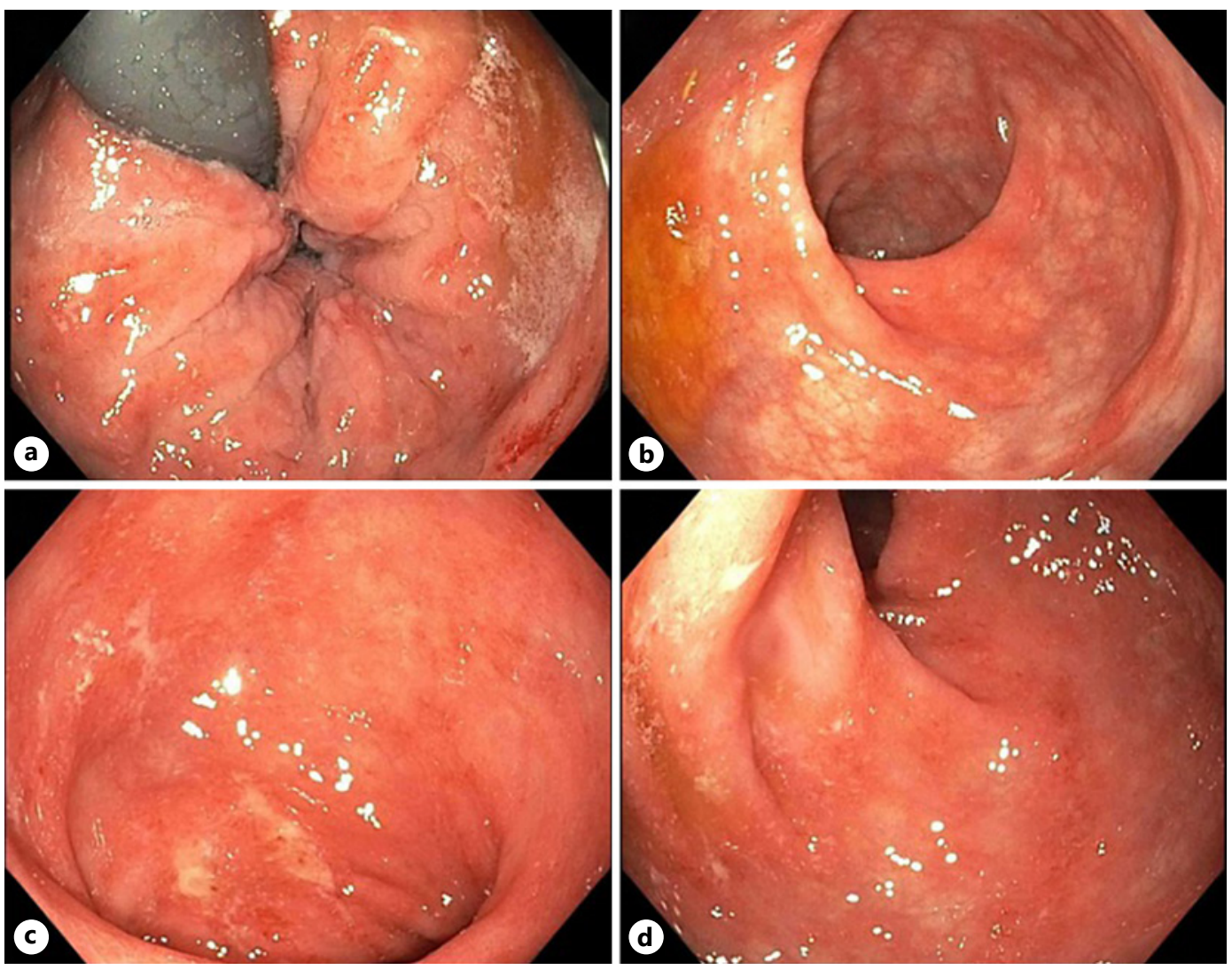

Fig. 2. Colonoscopy showed moderate diffuse inflammation characterized by altered vascularity, erythema, and granularity in the rectum (a), rectosigmoid (b), and sigmoid colon (c, d).

Hydrogen peroxide colitis results from highly reactive oxygen species, which causes damage to the colonic mucosa lining. Complications of hydrogen peroxide colitis include mild inflammatory changes, ulcers, stricture, colonic wall perforation, fistula formation, peritonitis, and even death.

Management is mostly conservative and includes discontinuation of exposure to the toxic age, fluid resuscitation, broad-spectrum antibiotics, and bowel rest. In severe cases, 5-aminosalicylic acid and corticosteroids can be used [18].

Early diagnosis is pertinent in preventing complications in these patients. There is a need to ask relevant questions regarding the history of colon-cleansing agents used in patients with acute onset of unexplained colitis. Early discontinuation of the toxic agent can lead to a decrease in morbidity and mortality. There is also a need to increase awareness among physicians and patients regarding the use of over-the-counter coloncleansing agents, leading to colitis. Patients with IBS should be advised to restrain from using a colon-cleansing agent without advice from their primary doctor as it can lead to various complications.

\section{Statement of Ethics}

Written informed consent was obtained from the patient for the publication of this case report. 
Mehershahi et al.: Colitis

\section{Conflict of Interest Statement}

The authors have no conflicts of interest to disclose.

\section{Funding Sources}

There was no external source of funding to support this case report.

\section{Author Contributions}

All the authors were involved in writing and finalizing the manuscript.

\section{References}

1 Vazquez Roque M, Bouras EP. Epidemiology and management of chronic constipation in elderly patients. Clin Interv Aging. 2015 Jun 2;10:919-30. eCollection 2015.

2 Choung RS, Locke GR, Schleck CD, Zinsmeister AR, Talley NJ. Cumulative incidence of chronic constipation: a population-based study 1988-2003. Aliment Pharmacol Ther. 2007;26(11-12):1521-8.

3 Hungin AP, Whorwell PJ, Tack J, Mearin F. The prevalence, patterns and impact of irritable bowel syndrome: an international survey of 40,000 subjects. Aliment Pharmacol Ther. 2003 Mar 1;17(5):643-50.

4 Camilleri M. Peripheral mechanisms in irritable bowel syndrome. N Engl J Med. 2012 Oct 25;367(17):162635.

5 Song GH, Venkatraman V, Ho KY, Chee MW, Yeoh KG, Wilder-Smith CH. Cortical effects of anticipation and endogenous modulation of visceral pain assessed by functional brain MRI in irritable bowel syndrome patients and healthy controls. Pain. 2006 Dec 15;126(1-3):79-90. Epub 2006 Jul 18.

6 Schmidt T, Hackelsberger N, Widmer R, Meisel C, Pfeiffer A, Kaess H. Ambulatory 24-hour jejunal motility in diarrhea-predominant irritable bowel syndrome. Scand J Gastroenterol. 1996 Jun;31(6):581-9.

7 Agrawal A, Houghton LA, Reilly B, Morris J, Whorwell PJ. Bloating and distension in irritable bowel syndrome: the role of gastrointestinal transit. Am J Gastroenterol. 2009 Aug;104(8):1998-2004. Epub 2009 Jun 2.

8 Chey WY, Jin HO, Lee MH, Sun SW, Lee KY. Colonic motility abnormality in patients with irritable bowel syndrome exhibiting abdominal pain and diarrhea. Am J Gastroenterol. 2001 May;96(5):1499-506.

9 Dominitz JA, Eisen GM, Baron TH, Goldstein JL, Hirota WK, Jacobson BC, et al. Complications of colonoscopy. Gastrointest Endosc. 2003 Apr 1;57(4):441-5.

10 Masannat YA, Harron M, Harinath G. Nonsteroidal anti-inflammatory drugs-associated colopathy. ANZ J Surg. 2010 Jan;80(1-2):96-9.

11 Mandzhieva B, Khan M, Rashid MU, Shobar R, Khan AH. Hydrogen peroxide enema-induced proctitis in a young female: a case report. Cureus. 2019 Dec 26;11(12):e6468.

12 Brun-Strang C, Dapoigny M, Lafuma A, Wainsten JP, Fagnani F. Irritable bowel syndrome in France: quality of life, medical management, and costs: the Encoli study. Eur J Gastroenterol Hepatol. 2007 Dec;19(12):1097103.

13 Mönnikes H. Quality of life in patients with irritable bowel syndrome. J Clin Gastroenterol. 2011 Aug; 45(Suppl): S98-S101.

14 Zhu L, Huang D, Shi L, Liang L, Xu T, Chang M, et al. Intestinal symptoms and psychological factors jointly affect quality of life of patients with irritable bowel syndrome with diarrhea. Health Qual Life Outcomes. 2015 Apr 18;13:49.

15 Böhn L, Störsrud S, Liljebo T, Collin L, Lindfors P, Törnblom H, et al. Diet low in FODMAPs reduces symptoms of irritable bowel syndrome as well as traditional dietary advice: a randomized controlled trial. Gastroenterology. 2015 Nov;149(6):1399-e2. Epub 2015 Aug 5.

16 Johannesson E, Simrén M, Strid H, Bajor A, Sadik R. Physical activity improves symptoms in irritable bowel syndrome: a randomized controlled trial. Am J Gastroenterol. 2011 May;106(5):915-22. Epub 2011 Jan 4.

17 Singh-Ranger G, Halls A, Grundy A, Kumar D. An unusual case of severe colitis after colonoscopy. J Crohns Colitis. 2011 Jun;5(3):267-8. Epub 2011 Mar 24.

18 Sheibani S, Gerson LB. Chemical colitis. J Clin Gastroenterol. 2008 Feb;42(2):115-21. 